\title{
Acute Respiratory Distress Syndrome during Pregnancy and Post-Partum - A Case Series with Spectrum of Near Miss to Mortality
}

\author{
Shruti Salampuria ${ }^{1}$, Shubhada Jajooz, Sourya Acharya ${ }^{3}$ \\ 1Department of Obstetrics and Gynaecology, Datta Meghe Institute of Medical Sciences, Sawangi (Meghe), \\ Maharashtra, India. ${ }^{2}$ Department of Obstetrics and Gynaecology, Datta Meghe Institute of Medical Sciences, Sawangi \\ (Meghe), Maharashtra, India. ${ }^{3}$ Department of General Medicine, Datta Meghe Institute of Medical Sciences, Sawangi \\ (Meghe), Maharashtra, India.
}

\section{INTRODUCTION}

Acute Respiratory Distress Syndrome is characterised by acute onset dyspnoea, hypoxemia, and diffuse pulmonary infiltrates. Many physiological changes occurring in pregnancy predispose to its occurrence. Maternal and perinatal mortality is high with significant morbidity in residual cases. Management strategies in obstetric population have been derived from general non-obstetric cases. Mechanical ventilation, both invasive and non-invasive, play a key role in treatment. In our case series, late identification of infection and postponement in referral were important factors contributing to maternal mortality. Many factors like pre-eclampsia, viral pneumonia, bacterial pneumonia, UTI lead to development of ARDS in pregnancy. ARDS leading to Multi-Organ Dysfunction Syndrome is an important cause of Maternal Mortality. Early detection of ARDS helps in improving both maternal and neonatal outcome. ARDS management depends on identifying the underlying cause and treating it along with maintaining the oxygen saturation. Mechanical Ventilation plays an important role in ARDS management. Multidisciplinary team approach by obstetricians, pulmonologists, intensive care providers is required. With the decrease in other causes of maternal death, mortality in obstetric patients due to ARDS is increasing significantly and needs to be addressed. It is a morbid condition, aetiology being inflammatory disease of lung that causes acute onset hypoxia due to noncardiogenic pulmonary oedema leading to respiratory failure. Bernard et al. and the American-European Consensus Conference proposed the present definition of ARDS. The criteria are- 1 ) acute onset 2) a $\mathrm{PaO}_{2} / \mathrm{FiO}_{2}$ ratio, or hypoxia score of $\leq 200$, irrespective of positive end expiratory pressure 3) bilateral infiltrates on chest X-ray and 4 ) a pulmonary artery occlusion pressure of $\leq 18 \mathrm{mmHg}$ or the absence of clinical evidence of left atrial hypertension.(1) Normal physiological changes occurring during pregnancy like increased vulnerability to infection, high chance of aspiration, reduced chest compliance, reduced functional residual capacity, mucosal oedema, reduced arterial $\mathrm{PaCO}_{2}$ predispose to ARDS and also make it difficult to manage.(2) Here we present cases of pregnant women in their peripartum period with sudden onset breathlessness and hypoxia caused by obstetric and non-obstetric causes with their clinical course, management and outcome. The women were treated at a rural tertiary care centre in Central India.

\section{KEY WORDS}

ARDS, Pregnancy, Maternal Mortality.
Corresponding Author: Dr. Shruti Salampuria, Room No. G-5, Shalinta Girls Hostel, Sawangi (Meghe), Wardha, Maharashtra, India.

E-mail: snsalampuria@gmail.com

DOI: $10.14260 / j e m d s / 2019 / 806$

Financial or Other Competing Interests: None.

How to Cite This Article:

Salampuria S, Jajoo S, Acharya S. Acute respiratory distress syndrome during pregnancy and post-partum- a case series with spectrum of near miss to mortality. J. Evolution Med. Dent. Sci. 2019;8(49):37243726, DOI: 10.14260/jemds/2019/806

Submission 20-09-2019,

Peer Review 15-11-2019,

Acceptance 23-11-2019,

Published 09-12-2019. 


\section{PRESENTATION OF CASE}

\section{Case 1}

A 22-year old primigravida, 35 weeks gestational age, came with complaints of fever and cough with expectoration since 3 days. On admission, she was febrile $\left(101^{\circ} \mathrm{F}\right)$, with tachycardia (Pulse- 102/min), tachypnoea (38 cycles/min), blood pressure was normal $(110 / 60 \mathrm{mmHg})$. On auscultation she had bilateral crepitations. $\mathrm{SpO}_{2}$ was $88 \%$ on room air. Obstetric findings were corresponding to gestational age and Non Stress Test was reactive. Medicine consultation was taken. Routine investigations showed no abnormalities. Total Leukocyte count was within normal limit. ABG showed hypoxia score $\left(\mathrm{PaO}_{2} / \mathrm{FiO}_{2}\right)$ as 188 . Chest X-ray (with shield) showed bilateral infiltrates (Figure 1). She was shifted to Medicine ICU. Sputum Culture showed no growth. Blood culture was also negative for any growth. H1N1 throat swab was negative. She was given propped up position and started on broad spectrum parenteral antibiotics. Patient was on oxygen by mask on Day 1 . As she was not maintaining saturation, was shifted to non-invasive ventilation on BiPAP from day 2 onwards for 3 days. Her breathlessness gradually reduced and $\mathrm{SpO}_{2}$ improved to $94 \%$ on room air on Day 5 . She was shifted to antenatal ward after 5 days. Rest of her antenatal period was uneventful. The pregnancy continued till term and had a favourable neonatal outcome.

\section{Case 2}

A 30-year-old, post-natal patient with pre-eclampsia who had parity score of 3 with all normal vaginal deliveries was on tab labetalol $100 \mathrm{mg}$ TDS since her $8^{\text {th }}$ month of antenatal period. On day 3 of delivery, she developed acute onset breathlessness. She had tachycardia (pulse- 116/min), blood pressure was $160 / 100 \mathrm{mmHg}$, tachypnoea (respiratory rate44 cycles/min), $\mathrm{SpO}_{2}$ was $86 \%$ on room air. On auscultationbilateral crepitations were present. Blood investigations (CBC, LFT, KFT, coagulation profile) were normal. Chest X-ray showed bilateral infiltrate (Figure 2). $\mathrm{ABG}$ showed $\mathrm{PaO}_{2}$ / $\mathrm{FiO}_{2}=170$. She was immediately shifted to MICU. Was started on non-invasive ventilation by BiPAP. She was given parenteral antibiotics, diuretics and antihypertensives. Her condition gradually improved with settlement of dyspnoea and control of hypertension in due course of 7 days. Review chest Xray done (Figure 3) was corresponding with relief of symptoms. She was later shifted to post-natal ward.

\section{Case 3}

A 20 -year-old primigravida, 22 weeks gestational age had history of fever since 1 week and breathlessness since 2 days. She was being treated at a private facility with antibiotics and antipyretics. Due to non-response to treatment she was referred to our tertiary care facility. On admission, she was anxious, febrile $\left(102^{\circ} \mathrm{F}\right)$, pulse- $120 / \mathrm{min}$, Blood pressure$90 / 60 \mathrm{~mm}$ of $\mathrm{Hg}$, respiratory rate-38 cycles/min. She had bilateral crepitations on auscultation. $\mathrm{SpO}_{2}$ was $80 \%$ on room air. Her blood investigations showed: Total Leukocyte count was $24,000 / \mathrm{mm}^{3}$ with $90 \%$ neutrophils and $15 \%$ band forms. Her chest X-ray (with shield) revealed bilateral infiltrates (Figure 4). Her hypoxia score was 158. She was shifted immediately to MICU and was put on NIV BiPaP. Blood culture showed growth of streptococcus pyogenes. Parenteral broad spectrum antibiotics were started. As she was not maintaining saturation on BiPAP, was intubated on the same day and put on ventilator and ventilated as per ARDS protocol. Fetal heart rate was regular around 120 beats $/ \mathrm{min}$. The general condition worsened. The patient developed septic shock on day 2 of admission and ionotropic support was started. The patient's condition deteriorated, and she succumbed.

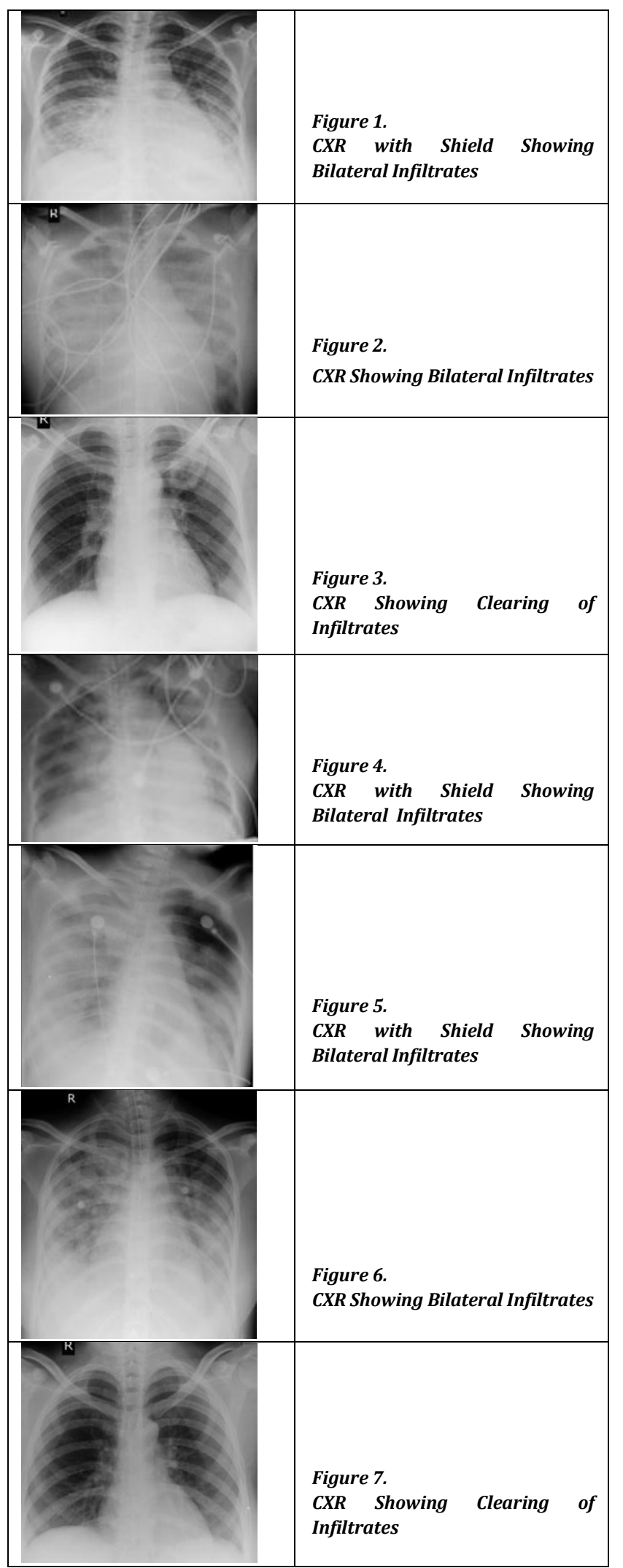




\section{Case 4}

A 28-year-old Gravida 2, 39 weeks gestational age, previous normal vaginal delivery, came to casualty with complaints of cough with expectoration since 3-4 days and difficulty in breathing since 1 day. She had history of fever with chills since 1 week for which she was being treated at a subcentre. On admission she was febrile $\left(100^{\circ} \mathrm{F}\right)$, had tachycardia (pulse $122 / \mathrm{min}$ ) and tachypnoea (RR- 44 cycles $/ \mathrm{min}$ ). $\mathrm{SpO}_{2}$ was $77 \%$ on room air. Bilateral crepitations were present. Chest Xray (with shield) showed bilateral infiltrates (Figure 5). ABG showed $\mathrm{PaO}_{2} / \mathrm{FiO}_{2}=150$. Her blood investigations depicted Total Leukocyte Count- $18,000 / \mathrm{mm}^{3}$. She was intubated and ventilated with ARDS protocol and was started on parenteral broad-spectrum antibiotics. She developed thrombocytopenia and coagulopathy (Platelet $=40,000 / \mathrm{mm}^{3}$, INR= 2.8) with deranged liver function test and renal function test. Fetal heart sound was absent on abdominal doppler and in view of disseminated intravascular coagulation and multiorgan dysfunction decision to terminate pregnancy was taken. The patient was induced, and she delivered an IUD foetus. Her general condition worsened on day 2 and was put on ionotropic support. She had sudden cardiac arrest and couldn't be revived. Was diagnosed as ARDS with Multi Organ Dysfunction Syndrome?

\section{Case 5}

A 30 year old P2L2, Day 2 LSCS was referred from a private setup, in view of increasing breathlessness. She had complaints of fever with chills since 5 days and burning micturition and was being managed conservatively. She had to be taken emergency LSCS in view of foetal distress. On $2^{\text {nd }}$ post-operative day she was dyspnoeic which progressed gradually and was transferred to our tertiary care facility. Her examination findings on admission were- febrile $\left(100^{\circ} \mathrm{F}\right)$, tachycardia (pulse rate- $110 / \mathrm{min}$ ), Respiratory rate was 30 cycles/min. Bilateral crepitations on auscultation were present. $\mathrm{SpO}_{2}$ was $86 \%$ on room air. Chest X-ray showed bilateral infiltrates (Figure 6). ABG showed hypoxia score of 190. Her Urine culture showed growth of Acinetobacter species. She was given propped up position and $\mathrm{O}_{2}$ by mask. There was no improvement in her oxygen saturation so was started on NIV BiPAP. She was administered parenteral antibiotics and bronchodilator nebulisation. The symptoms improved in 3 days and was weaned of BiPAP and shifted to $\mathrm{O}_{2}$ by nasal prongs. She was shifted to ward on post-operative day 10. Patient was discharged on Day 15 maintaining saturation on room air. On discharge review chest X-ray was done showed clearing of infiltrates. (Figure 7)

\section{DISCUSSION}

Early detection of ARDS helps in improving both maternal and neonatal outcome. Minor respiratory complaints should not be overlooked. Early evaluation and prompt treatment should be initiated. Underlying cause should be identified. Treatment depends upon the cause. In our case series, late identification of infection and postponement in referral were important factors contributing to maternal mortality. Chest $\mathrm{X}$ ray is an important modality in diagnosing ARDS and should not be delayed in pregnant patients with fear of radiation exposure to the foetus. Shielding of abdomen prevents the same. Maternal sepsis is a significant contributor to ICU admissions and maternal mortality.(3) One of the most important causes of maternal mortality in ARDS has been Multiple Organ Dysfunction Syndrome.(4) Pregnancy predisposes to development of ARDS by the following mechanisms-increased level of acute inflammatory reactants (ceruloplasmin, fibrinogen, plasminogen activator inhibitor-1), leukocytes and IL-12,(5) increased circulating blood volume, increased capillary leak and decreased serum albumin level.(6) The causes of ARDS are associated with obstetric causes such as preeclampsia, HELLP, tocolytic induced pulmonary oedema, amniotic fluid embolism, chorioamnionitis, septic abortion, endometritis and retained products of conception or nonobstetric causes that include sepsis, bacterial pneumonia, viral pneumonia, aspiration pneumonitis, TRALI, venous air embolism, malaria and trauma. Most common cause of ARDS in both obstetric and non-obstetric patients is sepsis with hypotension.(7) Neonatal outcomes in ARDS include intrauterine demise, perinatal hypoxia, preterm labour, and foetal heart rate abnormalities. Maternal $\mathrm{SaO}_{2}$ of around $95 \%$ which corresponds with foetal $\mathrm{PaO}_{2}$ of $70 \mathrm{mmHg}$ is required for adequate oxygenation.(7) Vigilant foetal monitoring should be done and decision of terminating pregnancy and mode of delivery should be decided depending upon the maternal and foetal status.

\section{REFERENCES}

[1] Bernard GR, Artigas A, Brigham KL, et al. The AmericanEuropean Consensus Conference on ARDS. Definitions, mechanisms, relevant outcomes and clinical trial coordination. Am J Respir Crit Care Med 1994;149(3 Pt 1):818-24.

[2] Vasudeva A, Bhat RG, Ramachandran A, et al. Infection and acute respiratory distress syndrome during pregnancy: a case series of preventable maternal deaths from southern India. J Infect Public Health 2013;6(1):55-7.

[3] Kankuri E, Kurki T, Carlson P, et al. Incidence, treatment and outcome of peripartum sepsis. Acta Obstet Gynecol Scand 2003;82(8):730-5.

[4] Catanzarite V, Willms D, Wong D, et al. Acute respiratory distress syndrome in pregnancy and the puerperium: causes, courses and outcomes. Obstet \& Gynecol 2001;97(5 Pt 1):760-4.

[5] Romero R, Gotsch F, Pineles B, et al. Inflammation in pregnancy: its roles in reproductive physiology, obstetrical complications and foetal injury. Nutr Rev 2007;65:S194-202.

[6] Lapinsky SE. Management of acute respiratory failure in pregnancy. Semin Respir Crit Care Med 2017;38(2):2017.

[7] Cole DE, Taylor TL, McCullough DM, et al. Acute respiratory distress syndrome in pregnancy. Crit Care Med 2005;33(Suppl 10):S269-78. 